\title{
Artifacts suppression in biomedical images using a guided filter
}

Bukreeva, I., Ingacheva, A., Fratini, M., Cedola, A., Junemann, O., et al.

I. Bukreeva, A. Ingacheva, M. Fratini, A. Cedola, O. Junemann, E. Longo, F. Wilde, J. Moosmann, A. Buzmakov, Y. Krivonosov, D. Zolotov, S. Saveliev, V. Asadchikov, M. Chukalina, "Artifacts suppression in biomedical images using a guided filter," Proc. SPIE 11605, Thirteenth International Conference on Machine Vision, 116050S (4 January 2021); doi: 10.1117/12.2587571

EDIE Event: Thirteenth International Conference on Machine Vision, 2020, Rome, Italy 


\title{
Artifacts suppression in biomedical images using a guided filter
}

\author{
I. Bukreeva ${ }^{1,2}$, A. Ingacheva ${ }^{3,4}$, M. Fratini ${ }^{1,5}$, A. Cedola ${ }^{1}$, O. Junemann ${ }^{6}$, E. Longo ${ }^{7}$, F. Wilde ${ }^{7}$, \\ J. Moosmann ${ }^{7}$, A. Buzmakov ${ }^{3}$, Y. Krivonosov' ${ }^{3}$, D. Zolotov ${ }^{3}$, S. Saveliev ${ }^{6}$, V. Asadchikov ${ }^{2}$ and \\ M. Chukalina ${ }^{3,4}$ \\ ${ }^{1}$ Institute of Nanotechnology- CNR, Rome Unit, Piazzale Aldo Moro 5, Italy \\ ${ }^{2}$ P.N. Lebedev Physical Institute, RAS, Leninskiy pr., 53 Moscow, Russian Federation \\ ${ }^{3}$ FSRC «Crystallography and photonics» RAS, Leninskiy pr., 59, Moscow, Russian Federation \\ ${ }^{4}$ Smart Engines Service LLC, 60-letiya Oktyabrya pr., 9, Moscow, Russian Federation \\ ${ }^{5}$ Fondazione Santa Lucia, Via Ardeatina 352, Roma, Italy \\ ${ }^{6}$ FSSI Research Institute of Human Morphology, Tsyurupy Str 3, Moscow, Russian Federation \\ ${ }^{7}$ Institute of Materials Research, Helmholtz-Zentrum Geesthacht, Max-Planck-Str. 1, 21502 \\ Geesthacht, Germany.
}

\begin{abstract}
Despite significant progress in computer vision, pattern recognition, and image analysis, artifacts in imaging still hampers the progress in many scientific fields relying on the results of image analysis. We here present an advanced image-based artifacts suppression algorithm for high-resolution tomography. The algorithm is based on guided filtering of a reconstructed image mapped from the Cartesian to the polar coordinates space. This postprocessing method efficiently reduces both ring- and radial streak artifacts in a reconstructed image. Radial streak artifacts can appear in tomography with an off-center rotation of a large object over 360 degrees used to increase the reconstruction field of view. We successfully applied the developed algorithm for improving $\mathrm{x}$-ray phase-contrast images of human post-mortem pineal gland and olfactory bulbs.
\end{abstract}

Keywords: guided filter, orthotropic artifacts, ring artifacts, radial streak artifacts, polar coordinates, pineal gland, olfactory bulb.

\section{INTRODUCTION}

Computer vision, pattern recognition, and image analysis for biomedical imaging applications have developed rapidly in recent decades and have played an important role in image registration, image retrieval, image processing, image analysis, and interpretation. These techniques help to efficiently retrieve relevant information in images. However, accurate results largely depend on the quality of the original images.

Synchrotron tomography is an effective tool for high-resolution 3D visualization of the interior structure of a wide variety of objects, including biological samples. Image acquisition methods have improved significantly over recent decades, and, nowadays, $x$-ray tomography is capable of reaching the micro- and nano-scale [1-3].

For the analysis of soft-tissue structures such as the brain, biomedical applications require apart from the high spatial resolution, the possibility of low-absorbing tissue visualization with a high image contrast [4]. X-ray phasecontrast tomography (XPCT) is an advanced imaging technique particularly adapted for soft biological tissue visualization. XPCT uses information about X-ray attenuation or/and phase changes in the object to reconstruct its interior structure.

Tomographic images of both high- and low- x-ray absorbing tissues can be affected by artifacts, and the image quality might significantly degenerate if image artifacts overlay the sample structural information [5, 6]. Artifacts suppression in tomographic reconstructions is generally performed with pre-processing algorithms. However, the pre-processing step does not always effectively remove artifacts [7, 8]; moreover, it might introduce new artifacts and distortions [8]. Thus post-processing procedure should be considered as an essential step for image reconstruction.

Thirteenth International Conference on Machine Vision, edited by Wolfgang Osten, Dmitry Nikolaev, Jianhong Zhou Proc. of SPIE Vol. 11605, 116050S · (c) 2021 SPIE · CCC code: 0277-786X/21/\$21 · doi: 10.1117/12.2587571 
A number of algorithms for ring and streak artifacts correction have been developed and implemented in computed tomography, in particular, as a post-processing step with an image mapped from the Cartesian to the polar coordinates space [9-14].

The aim of this study was to propose an effective post-processing method to reduce both the circle- and radial streak artifacts in computed tomography, specifically, in XPCT imaging with projections collected according to the so-called half acquisition mode (i.e., an off-center rotation of a large object over 360 degrees in order to increase the effective width of the field-of-view of the detector). The algorithm is based on a guiding filtering of the reconstructed image. The developed filter operates in the polar coordinates space, where ring- and radial streak artifacts would be represented as families of parallel stripes. The stripes corresponding to ring- and radial streak artifacts are perpendicular to each other and parallel to polar axes. Therefore the strategy of orthotropic artifacts suppression [15] is an appropriate approach to improve the image quality. These facts permitted to greatly simplify the task and helped to develop an efficient filter for artifacts suppression. The developed algorithm for artifacts removal was applied to the XPCT images of human post-mortem pineal gland and olfactory bulbs.

\section{GUIDED FILTER FOR ARTIFACTS SUPPRESSION IN XPCT IMAGING EXPERIMENT}

We propose an effective image method to reduce both ring- and radial streak artifacts in tomographic images. We applied our algorithm for X-ray phase-contrast tomography (XPCT) of post-mortem human pineal gland and olfactory bulb.

\subsection{Samples}

We investigated three post-mortem human samples: two olfactory bulbs and one pineal gland. The study was carried out on autopsy material obtained from the collection of Federal State Scientific Institution Research Institute of Human Morphology (Moscow, Russian Federation). All protocols were approved by the Ethical Committee of the Research Institute of Human Morphology of the Russian Academy of Medical Sciences (now FSSI Research Institute of Human Morphology) (No. 6A of October 19, 2009) and are in correspondence with instructions of the Declaration of Helsinki including points 7-10 for human material from 12.01.1996 with the last amendments from 19.12.2016.

\subsection{XPCT experiment and phase reconstruction}

Inline XPCT is a phase-sensitive X-ray imaging technique providing 3D visualization with enhanced soft-tissue contrast. The data were acquired at the P05 beamline of the synchrotron facility PETRA III, DESY, operated by the Helmholtz-Zentrum Geesthacht [16].

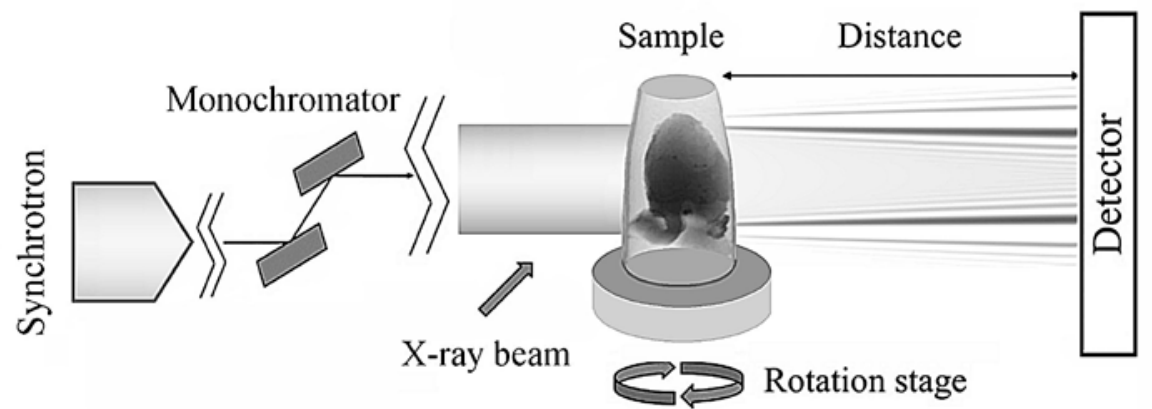

Figure1. A schematic view of the phase-contrast tomography experiment as used at beamline P05, PETRA III, DESY, Hamburg, Germany, operated by Helmholtz-Zentrum Geesthacht. The sample was placed downstream of the monochromator. The detector was placed at a near-field distance of $0.5 \mathrm{~m}$ from the sample. The sample was rotated by 360 degrees with stepwise rotation and

The setup of the experiment is schematically shown in Fig.1. An object was illuminated with monochromatic Xray beam with energy $25 \mathrm{keV}$. The sample was mounted on a stage supplying its translation and rotation. The CMOS (KIT) detector was placed at a near-field distance with a sample-detector distance of $0.5 \mathrm{~m}$. Effective pixel size was about of 1.28x1.28 micron ${ }^{2}$, field of view of $6.56 \times 4.92 \mathrm{~mm}^{2}$, and an exposure time of $70 \mathrm{~ms}$ for each projection.

The object was rotated by 360 degrees with stepwise rotation, and projections were collected according to the half acquisition mode [17]. This detection method is based on an off-center rotation of the sample over 360 degrees. 
Usually, half acquisition mode is used when the projected image of the object does not fit entirely in the field of view of the detector (FOV). The FOV can be artificially increased by rotating the object over 360 degrees.

4000 projections were acquired per scan. The pixel size was $1.28 \times 1.28$ micron $^{2}$.

The raw data were pre-processed with the flat-/dark-fields correction and a phase-contrast retrieval was applied to each projection:

$$
T=\frac{2 \pi}{\lambda z}\left(F^{-1}\left\{\frac{F\left[I(x, y, z=D) / I_{0}-1\right]}{\left(k_{x}^{2}+k_{y}^{2}+\alpha\right)}\right\}\right),
$$

where $\mathrm{T}$ is the retrieved image, $\alpha$ is a regularization parameter which regulates the trade-off between blurring and suppression of phase-contrast induced fringes, $\lambda$ is the wave length corresponding to the energy $E,\left(k_{x}, k_{y}\right)$ are spatial frequencies reciprocal to $(\mathrm{x}, \mathrm{y}), . \mathrm{I}_{0}$ is the incident intensity, however for practical reasons the intensity was measured at distance $\mathrm{D}$ without the sample and this is thus just an approximation of $\mathrm{I}_{0}$.

To obtain Eq.(1) we used a linearization of the Transport of Intensity equation with an additional regularization for the singularity at zero frequency. The set of projections Eq.(1) was utilized to reconstruct the 3D image of the sample with Filtered Back Projection method (FBP) using the tomographic reconstruction pipepline [18]. The reconstructed images of the human olfactory bulbs are shown in Figs.2a, 3a. Figure 4a represents the image of the human pineal gland with ring and radial streaks artifacts.
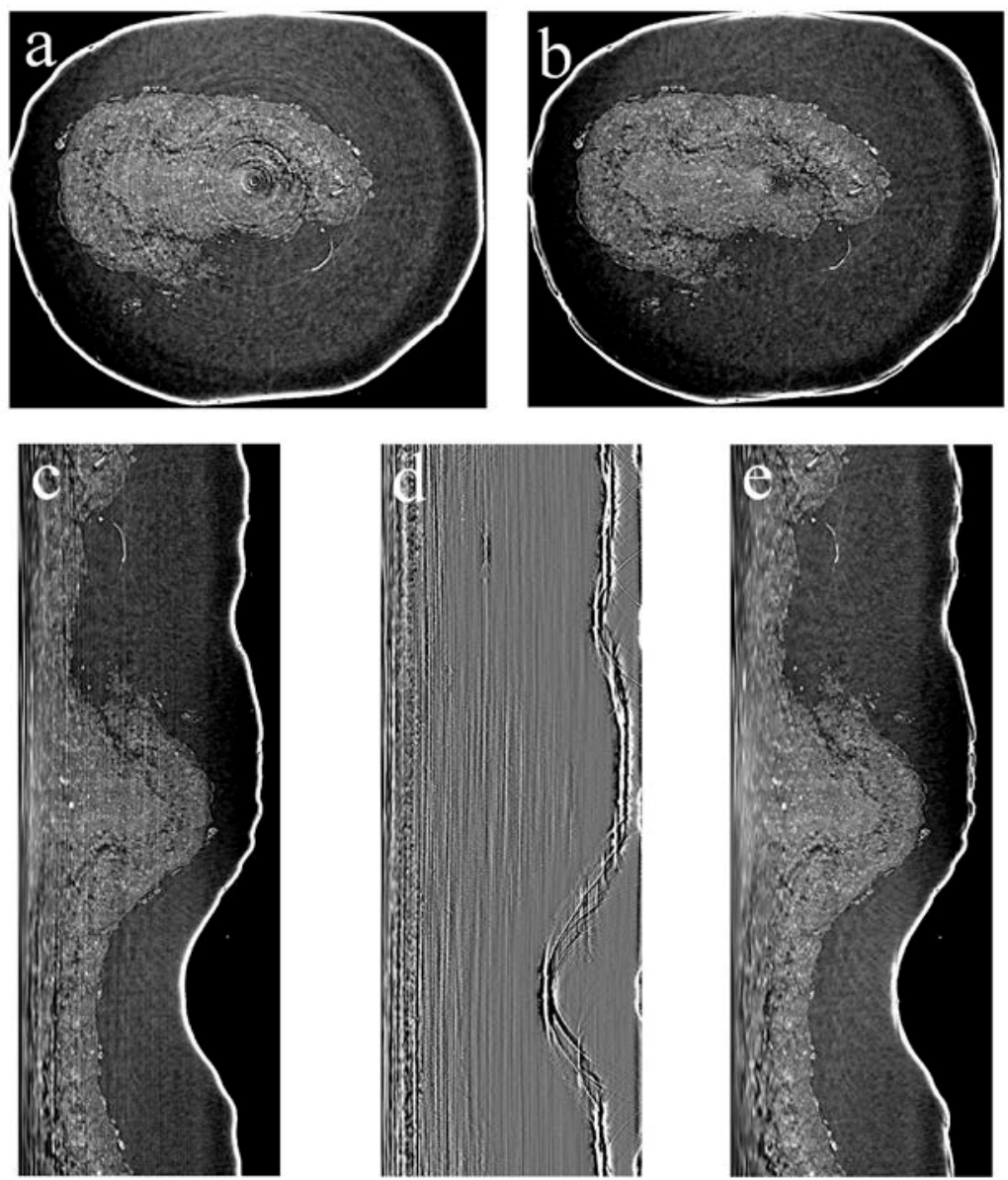

Figure 2. The reconstructed images of human olfactory bulb (a) image of the sample in the Cartesian coordinates shows the ring artifacts, (b) the same image as (a) after suppression of ring artifacts, (c-e) the polar coordinates: (c) image with ring artifacts visible as vertical gray strips, (d) ring artifacts, (e) image after the artifacts suppression. 

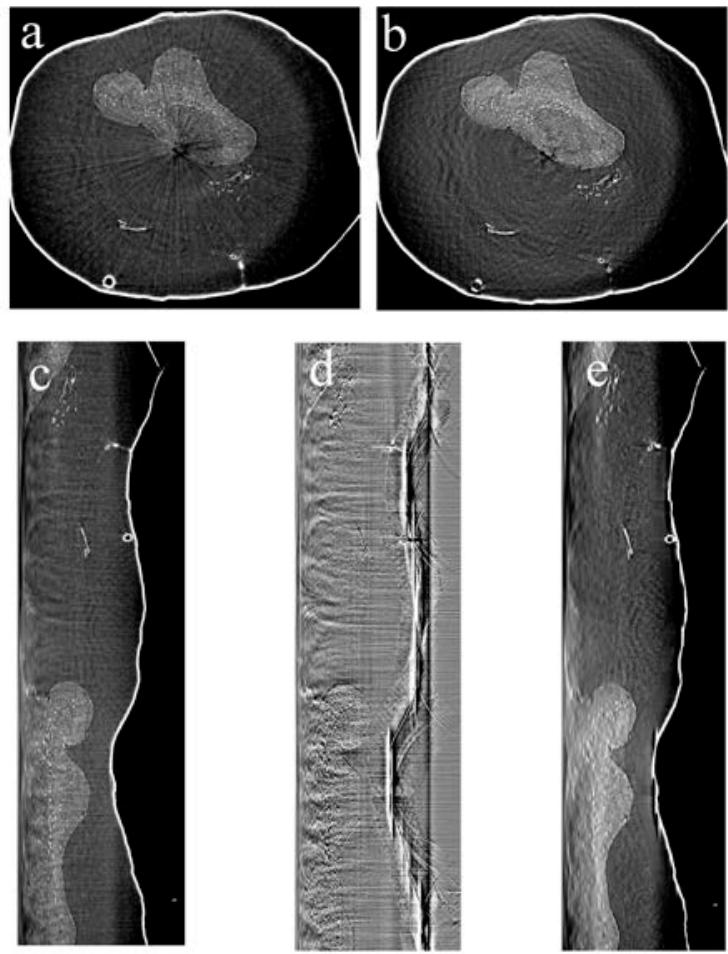

Figure 3. The reconstructed images of the human olfactory bulb (a-b) the Cartesian coordinates: (a) image of the sample with the radial streak artifacts, (b) the same image as (a) after suppression of radial streak artifacts, (c-e) the polar coordinates: (c) image with radial streak artifacts visible as horizontal stripes, (d) radial streak artifacts, (e) image after the artifacts suppression.
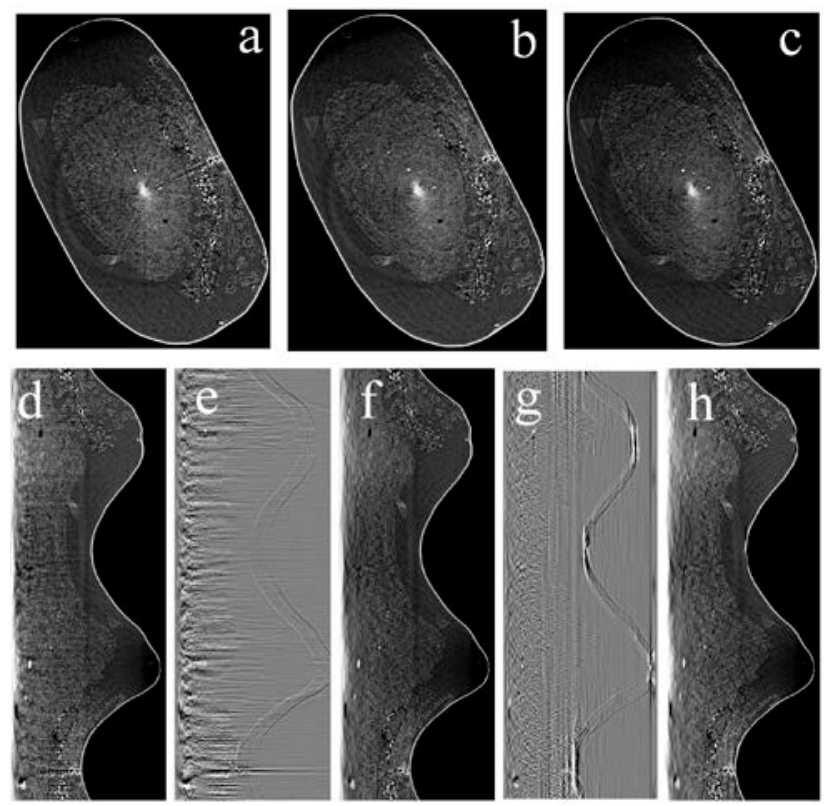

Figure 4. The reconstructed images of the human pineal gland (a-c) the Cartesian coordinates: (a) image of the sample with both ring- and radial streak artifacts; (b) the same image as in figure (a) after suppression of radial streak artifacts; (c) the same image as in figure (b) after suppression of ring artifacts; (d-h) the polar coordinates: (d) image with both streak- and ring artifacts visible as mutually perpendicular strips, (e) radial streak artifacts, (f) image after the radial streak artifacts suppression, (g) ring artifacts, (h) image after the rings artifact suppression. 


\subsection{Cartesian and polar coordinates converting}

As a first step, the developed algorithm converts the Cartesian coordinates to the polar coordinates:

$$
\begin{gathered}
\rho=\sqrt{\left(x-x_{c}\right)^{2}+\left(y-y_{c}\right)^{2}} \\
\theta=\tan ^{-1} \frac{y-y_{c}}{x-x_{c}},
\end{gathered}
$$

where the origin of the Cartesian space $\left(\mathrm{x}_{\mathrm{c}}, \mathrm{y}_{\mathrm{c}}\right)$ is set in the center of the image, $(\mathrm{x}, \mathrm{y})$ denotes the sampling pixel in the Cartesian coordinates, $(\rho, \theta)$ denotes the radius and the angular position in the polar coordinates.

One of the main properties of the Cartesian to polar coordinates transformation is a conversion of concentric rings and radial lines in the Cartesian coordinates into straight lines along the $\theta$ and $\rho$ axis directions in the polar coordinates, respectively.

\subsection{Filter for artifacts suppression in XPCT imaging of biological objects}

The algorithm proposed here might be regarded as guided filtering [19] in the polar space. Guided filtering uses the content of an auxiliary image (a guidance image) to perform the filtering procedure at the original (the guided) image. In our research, as a guided input, we used the tomographic images distorted with ring- and/or radial streak artifacts. The guidance images have been generated from the corresponding guided input such a way that the local contrast would not be distorted by artifacts. The main contribution to the filtered result comes from the pixels of the guided image with values close to the guidance image pixels. Thus, any low-frequency distortion of the guidance image practically does not affect the filtered output.

The proposed algorithm is based on the Cartesian-polar transformation of the image and in-line scale filtering. In the Cartesian coordinates, rings artifacts appear as a complete or incomplete concentric rings around the center of rotation of the tomographic setup. Ring artifacts mainly occur due to miscalibrated or defective detector elements. Figure 2a shows a ring distortion of the image after reconstruction. Streak artifacts shown in Figs. 3a,4a are visible as radial stripes running from the center of the image. In the polar coordinates, both ring- and radial streak artifacts would be converted to families of parallel stripes (in case of streak artifacts, the stripes have a tapering profile). Furthermore, the stripes corresponding to ring- and streak artifacts are mutually perpendicular. Therefore the problem of the artifacts can be solved with an orthotropic artifacts suppression method by guided filtering. To construct the guidance image, we calculated the derivative of the image in the horizontal direction (with finite difference method), i.e. in the direction perpendicular to the vertical stripes [20]. As a next step, for each column we performed one direction median filtering to reduce the contribution of the high-frequency noise component and applied the cumulative summation operation line by line resulting in an image preserving low-frequency components. The final guidance image was created by subtracting the result from the guided image. After the generation of the guidance image, we performed the guiding filtering operation. We used a local model between the guidance I and the filtered output q:

$$
q_{i}=a_{k} I_{i}+b_{k}, \forall i \in \omega_{k}
$$

where $\left(a_{k} b_{k}\right)$ are linear coefficients assumed constant in a window $\omega_{k}$ centered at the pixel $k$.

The coefficients $\left(a_{k}, b_{k}\right)$ can be found as a solution of the linear ridge regression model [21] and are given by the expressions

$$
\begin{aligned}
& \mathrm{a}_{\mathrm{k}}=\frac{\frac{1}{|\omega|} \sum_{\mathrm{i} \in \omega_{\mathrm{k}}} \mathrm{I}_{\mathrm{i}} \mathrm{p}_{\mathrm{i}}-\mu_{\mathrm{k}} \overline{\mathrm{p}_{\mathrm{k}}}}{\sigma_{\mathrm{k}}^{2}+\varepsilon} \\
& \mathrm{b}_{\mathrm{k}}=\overline{\mathrm{p}_{\mathrm{k}}}-a_{k} \mu_{k^{\prime}}
\end{aligned}
$$

where $\sigma_{k}^{2}$ and $\mu_{k}$ are the variance and the mean of $I$ in the window $\omega_{\mathrm{k}}$ respectively, $|\omega|$ is the number of pixels in $\omega_{\mathrm{k}}$, and $\overline{p_{k}}$ is the mean value of $p$ in $\omega_{\mathrm{k}}$, where $p$ is the input image. 
The final guiding output was found as

$$
q_{i}=\bar{a}_{\imath} I_{i}+\bar{b}_{\imath}
$$

where $\bar{a}_{l}$ and $\bar{b}_{l}$ are the average coefficients of all windows overlapping $i$.

The reconstructed image of the sample with suppressed artifacts was obtained as a result of the mapping of output Eq.(7) from polar to Cartesian coordinates.

\subsection{Suppression of radial streak artifacts}

As it was mentioned before, a concentric rings and radial lines in the Cartesian coordinates are mapped into straight lines along the $\theta$ and $\rho$ axis directions in polar coordinates, respectively. However if artifacts in the Cartesian coordinates are not lines but stripes, the mapping of the images to the polar coordinates space leads to the broadening of stripes profile in the direction of short $\rho$ (see images in the polar coordinates in Figs.2-3).

It takes place for the following reason. To prevent missing of image pixels at large radius $\rho=\mathrm{R}_{\max }$ the sampling in the angular direction should satisfy the next condition [22]:

$$
n_{\theta} \geq 2 \pi R_{\max }
$$

where $\mathrm{n}_{\theta}$ is the number of samples (points) in the angular direction. A square reconstructed image in the Cartesian coordinates is given with the size $2 \mathrm{R}_{\max } \times 2 \mathrm{R}_{\max }$ pixels.

This leads to oversampling of the image pixels at short distances $\rho$. Indeed, in order to sample radius $\rho$ evenly covers 360 degrees in angular direction for $\theta=0, \ldots \mathrm{n}_{\theta}$, the number of samples $\mathrm{n}_{\theta}$ should be significantly increased at short distances. Since no information is gained from oversampling area the number of samples in the angular direction should be adapted according to the size of $\rho$ [22].

We generated the series of images rescaled along $\theta$ axis in a step-like manner with the scaling factor $k$ increasing along $\theta$ axis from $1 / 12$ to 1 with step 1/3. This procedure helps us on the one hand to avoid the broadening of radial streak artifacts at short distances $\rho$ and on the other hand to prevent missing of image pixels at the large distance $R_{\max }$. For each scaled image, orthotropic artifacts were suppressed using the algorithm described in Ref [20]. Each image with suppressed artifacts was converted to the Cartesian coordinates. The central square part of the images in the Cartesian coordinates with the side length given by $n / 2^{1 / 2} k$ and satisfying the sampling condition was cut and inserted into the final output image.

\subsection{Artifacts suppression in biological samples}

The olfactory bulb images before- and after suppression of ring artifacts are shown in Figs.2a and 2b, respectively. Figures 2c,e represent the images of the samples with ring artifacts (vertical gray stripes) and with the suppressed artifacts, respectively, in the polar coordinates. Figure $2 \mathrm{~d}$ is the result of the subtraction of Fig.2c and Fig.2e. Figures 3a,b shows the olfactory bulb image with radial streak artifacts. Figures 3c,e are images with the radial streak artifacts (horizontal stripes) and without the artifacts, respectively, in the polar coordinates. Figure 3d illustrates the difference between Fig.3c and Fig.3e.

Figure 4 illustrates the process of the suppression of both ring and radial streak artifacts in the image of the human pineal gland. Figures 4a shows a reconstructed image with artifacts. Figures 4b,c are results after radial streak and ring artifacts suppression, respectively. Figures 4d-f illustrate the radial streak artifacts (horizontal stripes) suppression in the polar coordinates. Figures $4 \mathrm{f}$-h show the ring artifacts (vertical stripes) suppression in the polar coordinates.

\subsection{Computational platform}

Data pre-processing, phase retrieval, and FBP reconstruction were done with the software of beamline P05 at PETRA III provided by HZG [18] which uses the ASTRA toolbox [23] as backend for the tomographic backprojection. The guided filter for orthotropic artifacts suppression was developed by Smart Engines Service LLC [20]. 
3D rendering procedures were done using image processing program Fiji /ImageJ [24].

\section{CONCLUSION}

The ability to effectively suppress artifacts in tomography images is essential in many scientific fields, including biomedicine. To improve image quality, different methods and correction algorithms are included in a synchrotron beam line software package. In particularly, scans can be acquired with random lateral translations of the sample between projections (offered at P05), which is effective to reduce artifacts. This however, increases scan times and is not always a relevant option, especially for in situ or time-resolved scans or when the sample is prone to movements during the scan. Therefore pre-processing steps might not be sufficient and user would be engaged in problem of residual artifacts suppression in reconstructed images.

Here we propose the algorithm incorporated the guided filtering method in the polar space to suppression artifacts in a reconstructed tomographic image. The proposed algorithm is based on Cartesian-polar transformation that greatly simplified the task reducing the problem of ring- and radial streak artifacts removal in the Cartesian space to simple orthotropic artifacts suppression procedure in the polar coordinates space.

It was shown that proposed algorithm suppress the artifacts in XPCT reconstructed images of human postmortem pineal gland and olfactory bulbs and help to retrieve the lost information. As future work we plan to expand our approach to non-radial streak artifacts suppression. Application of vanishing points detectors [25] would enable to localize the center of streak artifacts and to rectify new center of the polar coordinate system.

\section{ACKNOWLEDGEMENTS}

The bilateral project CNR/RFBR (2018- 2020) - accordo CNR-RFBR delle Relazioni Internazionali (CUP B86C17000210005) \& Russian number 18-52-7819), the FISR Project "Tecnopolo di nanotecnologia e fotonica per la medicina di precisione” (funded by MIUR/CNR, CUP B83B17000010001) and the TECNOMED project (funded by Regione Puglia, CUP B84I18000540002) are acknowledged for financial support. This work was partially supported by RFBR (grant 18-29-26028). We also thank the Ministry of Science and Higher Education of Russian Federation within the State assignment FSRC «Crystallography and Photonics» RAS for financial supporting our work on tomography algorithms development. M. Fratini acknowledges the Italian Ministry of Health Young Researcher Grant 2013 (GR-2013-02358177) for financial support.

\section{REFERENCES}

[1] A. Cedola, A. Bravin, I. Bukreeva, M. Fratini, A. Pacureanu, A. Mittone, L. Massimi, P. Cloetens, P. Coan, G. Campi, R. Spanò, F. Brun, V. Grigoryev, V. Petrosino, C. Venturi, M. Mastrogiacomo, N. Kerlero De Rosbo, A. Uccelli, "X-Ray Phase Contrast Tomography Reveals Early Vascular Alterations and Neuronal Loss in a Multiple Sclerosis Model”, Sci. Rep., 7, 1-11, (2017); Online:. https://doi.org/10.1038/s41598-017-06251-7.

[2] A. Khimchenko, C. Bikis, A. Pacureanu, S.E. Hieber, P. Thalmann, H. Deyhle, G. Schweighauser, J. Hench, S. Frank, M. Müller-Gerbl, G. Schulz, P. Cloetens and B. Müller, "Hard X-Ray Nanoholotomography: LargeScale, Label-Free, 3D Neuroimaging beyond Optical Limit”, Adv. Sci., 5, 1700694, (2018); Online: http://doi.wiley.com/10.1002/advs.201700694.

[3] L. Massimi, I. Bukreeva, G. Santamaria, M. Fratini, A. Corbelli, F. Brun, S. Fumagalli, L. Maugeri, A. Pacureanu, P. Cloetens, N. Pieroni, F. Fiordaliso, G. Forloni, A. Uccelli, N. Kerlero de Rosbo, C. Balducci and A. Cedola, "Exploring Alzheimer's disease mouse brain through X-ray phase-contrast tomography: From the cell to the organ”, Neuroimage, 184, 490-5, (2019).

[4] M. Fratini, I. Bukreeva, G. Campi, F. Brun, G. Tromba, P. Modregger, D. Bucci, G. Battaglia, R. Spanò, M. Mastrogiacomo, H. Requardt, F. Giove, A. Bravin and A. Cedola, "Simultaneous submicrometric 3D imaging of the micro-vascular network and the neuronal system in a mouse spinal cord”, Sci. Rep., 5, 8514, (2015); Online: https://www.ncbi.nlm.nih.gov/pmc/articles/PMC4649670. 
[5] L. Gjesteby, B. De Man, Y. Jin, H. Paganetti, J. Verburg, D. Giantsoudi, and G. Wang, "Metal artifact reduction in CT: where are we after four decades?”, IEEE Access, 4, 5826-5849, (2016).

[6] I. Bukreeva, V. Asadchikov, A. Buzmakov, M. Chukalina, A. Ingacheva., F. Palermo, M. Fratini and A. Cedola, "Simultaneous iterative reconstruction method for high resolution x-ray phase-contrast tomography", Proc. SPIE, vol. 11433, Twelfth International Conference on Machine Vision (ICMV 2019); 114331V (2020); https://doi.org/10.1117/12.2557133.

[7] M. Zellerhoff, B. Scholz, E-P. Ruehrnschopf and T. Brunner, "Low contrast 3D reconstruction from C-arm data", Proc. SPIE, 5745, 646-55, (2005).

[8] T. Riess, T. Fuchs and W.A. Kalender, "A new method to identify and to correct circular artifacts in x-ray CT images”, Phys. Medica, 2, 43-55, (2004).

[9] J. Sijbers and A. Postnov, "Reduction of ring artefacts in high resolution micro-CT reconstructions," Phys. Med. Biol., 49, 14, N247-N253, (2004).

[10] X. Liang, Z. Zhang, T. Niu, S. Yu, S. Wu, Z. Li, H. Zhang, and Y. Xie, "Iterative image-domain ring artifact removal in cone-beam CT," Phys. Med. Biol., 62, 13, 5276-5292, (2017).

[11] H. Wu, J. Li, and H. Wang, "Removing ring artifacts in cone-beam CT via TV-Stokes and unidirectional total variation model,”' Med. Phys., 46, 4, 1719-1727, (2019).

[12] M. Johari, M. Abdollahzadeh, F. Esmaeili, and V. Sakhamanesh, "Metal Artifact Suppression in Dental Cone Beam Computed Tomography Images Using Image Processing Techniques”, J Med Signals Sens., 8, 1, 12-24, (2018).

[13] V. Naranjo, R. Lloréns, M. Alcañiz, F. López-Mir, "Metal artifact reduction in dental CT images using polar mathematical morphology”, Comput. Methods Programs Biomed, 102, 64-74, (2011).

[14] V. Naranjo, R. Llorens, P. Paniagua, M. Alcaniz, and S. Albalat, "A new approach in metal artifact reduction for CT 3D reconstruction," Bioinspired Applications in Artificial and Natural Computation, 11-19, (2009).

[15] A.V. Dolmatova, E. E. Berlovskaya, I. Bukreeva, A. Cedola, B. R. Islamov, E. Kuznetsova, I. A. Ozheredov, D. P. Nikolaev, "Orthotropic artifacts suppression for $\mathrm{THz}$ and $\mathrm{x}$-ray images using guided filtering", Proceedings Volume 11433, Twelfth International Conference on Machine Vision (ICMV 2019); 1143330 (2020); https://doi.org/10.1117/12.2557499

[16] F. Wilde, M. Ogurreck, I. Greving, J. U.Hammel, F. Beckmann, A. Hipp, L. Lottermoser, I. Khokhriakov, P. Lytaev, T. Dose, H. Burmester, M. Müller and A. Schreyer, "Micro-CT at the imaging beamline P05 at PETRA III” AIP Conference Proceedings vol 1741 (American Institute of Physics Inc.) p. 030035 (2016); Online: http://aip.scitation.org/doi/abs/10.1063/1.4952858.

[17] G. Wang, “X-ray micro-CT with a displaced detector array”, Med. Phys., 29, 1634-6, (2002).

[18] J. Moosmann, A. Ershov, V. Weinhardt, T. Baumbach, M. S. Prasad, C. LaBonne, X. Xiao, J. Kashef and R. Hofmann, "Time-lapse X-ray phase-contrast microtomography for in vivo imaging and analysis of morphogenesis,” Nature Protocols 9(2), 294-304 (2014); DOI: 10.1038/nprot.2014.033.

[19] K. He, J. Sun, and X. Tang, “Guided image filtering,” IEEE Trans. Pattern Anal. Mach. Intell., 35, 6, 13971409, (2013).

[20] E.E. Berlovskaya, A.V. Buzmakov, A.S. Ingacheva, A.M. Makurenkov, D.P. Nikolaev, I.A. Ozheredov, M.V. Chukalina, A.P. Skurinov, "Suppression algorithm for the orthotropic artifacts in images registered in X-ray and terahertz band”, Information Processes, 19, 2, 200-207, (2019) (in Russian).

[21] N. Draper and H. Smith, “Applied Regression Analysis”, second ed.John Wiley, (1981).

[22] R. Matungka, Y. F. Zheng, and R. L. Ewing, “Image Registration Using Adaptive Polar Transform”, IEEE transactions on image processing, 18, 10, (2019).

[23] van Aarle W. et al., "The ASTRA Toolbox: A platform for advanced algorithm development in electron tomography”, Ultramicroscopy, 157, 35-47 (2015).

[24] J. Schindelin et al., "Fiji: an open-source platform for biological-image analysis", Nature methods, 9, 676 (2012).

[25] A. Sheshkus, A. Ingacheva, and D. Nikolaev "Vanishing points detection using combination of fast Hough transform and deep learning", Proc. SPIE 10696, Tenth International Conference on Machine Vision (ICMV 2017), 106960H (13 April 2018); https://doi.org/10.1117/12.2310170. 